\title{
The Sorting Order on a Coxeter Group
}

\author{
Drew Armstrong \\ School of Mathematics, University of Minnesota, 206 Church St. SE, Minneapolis, MN 55455 USA
}

\begin{abstract}
Let $(W, S)$ be an arbitrary Coxeter system. For each sequence $\omega=\left(\omega_{1}, \omega_{2}, \ldots\right) \in S^{*}$ in the generators we define a partial order — called the $\omega$-sorting order—on the set of group elements $W_{\omega} \subseteq W$ that occur as finite subwords of $\omega$. We show that the $\omega$-sorting order is a supersolvable join-distributive lattice and that it is strictly between the weak and strong Bruhat orders on the group. Moreover, the $\omega$-sorting order is a "maximal lattice" in the sense that the addition of any collection of edges from the Bruhat order results in a nonlattice.
\end{abstract}

Along the way we define a class of structures called supersolvable antimatroids and we show that these are equivalent to the class of supersolvable join-distributive lattices.

Keywords: Coxeter group, join-distributive lattice, supersolvable lattice, antimatroid, convex geometry

\section{Extended Abstract}

Let $(W, S)$ be an arbitrary Coxeter system and let $\omega=\left(\omega_{1}, \omega_{2}, \ldots\right) \in S^{*}$ be an arbitrary sequence in the generators, called the sorting sequence. We will identify a finite subword $\alpha \subseteq \omega$ with the pair $(\alpha, I(\alpha))$, where $I(\alpha) \subseteq I(\omega)=\{1,2, \ldots\}$ is the index set encoding the positions of the letters. Given a word $\alpha=\left(\alpha_{1}, \ldots, \alpha_{k}\right) \in S^{*}$, let

$$
\langle\alpha\rangle=\alpha_{1} \cdots \alpha_{k} \in W
$$

denote the corresponding group element. The subsets of the ground set $I(\omega)$ are ordered lexicographically: if $A$ and $B$ are subsets of $I(\omega)$ we say that $A \leq_{\operatorname{lex}} B$ if the minimum element of $(A \cup B) \backslash(A \cap B)$ is contained in $A$.

Definition 1 We say that a finite subword $\alpha \subseteq \omega$ of the sorting sequence is $\omega$-sorted if

1. $\alpha$ is a reduced word,

2. $I(\alpha)=\min _{\leq_{\operatorname{lex}}}\{I(\beta) \subseteq I(\omega):\langle\beta\rangle=\langle\alpha\rangle\}$.

That is, $\alpha$ is $\omega$-sorted if it is the lexicographically-least reduced word for $\langle\alpha\rangle$ among subwords of $\omega$.

Let $W_{\omega} \subseteq W$ denote the set of group elements that occur as subwords of the sorting sequence. Then $\omega$ induces a canonical reduced word for each element of $W_{\omega}$-its $\omega$-sorted word. This, in turn, induces a partial order on the set $W_{\omega}$ by subword containment of sorted words.

Definition 2 Given group elements $u, w \in W_{\omega}$, we write $u \leq_{\omega} w$ if the index set of $\omega$-sort $(u)$ is contained in the index set of $\omega$-sort $(w)$. This is called the $\omega$-sorting order on $W_{\omega}$. 
The sorting orders are closely related to other important orders on the group.

Theorem 1 Let $\leq_{\mathrm{R}}$ denote the right weak order and let $\leq_{\mathrm{B}}$ denote the Bruhat order on $W$. For all $u, w \in W_{\omega}$ we have

$$
u \leq_{\mathrm{R}} w \Rightarrow u \leq_{\omega} w \Rightarrow u \leq_{\mathrm{B}} w .
$$

For example, let $W=\mathfrak{S}_{4}$ be the symmetric group of permutations of $\{1,2,3,4\}$ with the generating set of adjacent transpositions

$$
S=\left\{s_{1}=(12), s_{2}=(23), s_{3}=(34)\right\}
$$

Figure 1 displays the Hasse diagrams of the weak order, $\left(s_{1}, s_{2}, s_{3}, s_{2}, s_{1}, s_{2}\right)$-sorting order and strong order on the symmetric group $\mathfrak{S}_{4}$ - the weak order is indicated by the shaded edges; solid edges indicate the sorting order; solid and broken edges together give the Bruhat order.

It turns out that the collection of $\omega$-sorted words has a remarkable structure. Given a ground set $E$ and a collection of finite subsets $\mathscr{F} \subseteq 2^{E}$, the pair $(E, \mathscr{F})$ is called a set system. A set system $(E, \mathscr{F})$ is called an antimatroid (see (11)) if it satisfies

- For all nonempty $A \in \mathscr{F}$ there exists $x \in A$ such that $A \backslash\{x\} \in \mathscr{F}$,

- For all $A, B \in \mathscr{F}$ with $B \nsubseteq A$ there exists $x \in B \backslash A$ such that $A \cup\{x\} \in \mathscr{F}$.

Equivalently, $\mathscr{F}$ is the collection of open sets for a closure operator $\tau: 2^{E} \rightarrow 2^{E}$ that satisfies the anti-exchange property:

- If $x, y \notin \tau(A)$ then $x \in \tau(A \cup\{y\})$ implies $x \notin \tau(A \cup\{x\})$.

Such an operator $\tau$ models the notion of "convex hull", and so it is called a convex closure. Furthermore, we say that a lattice $L$ is join-distributive if it satisfies:

- For each $x \in L$, the interval $[x, y]$, where $y$ is the join of elements that cover $x$, is a boolean algebra.

Edelman (5) proved that a finite lattice is join-distributive if and only if it arises as the lattice of open sets of a convex closure. We will generalize Edelman's characterization to the case of supersolvable join-distributive lattices.

Definition 3 Consider a set system $(E, \mathscr{F})$ on a totally ordered ground set $\left(E, \leq_{E}\right)$. We say that $(E, \mathscr{F})$ is a supersolvable antimatroid if it satisfies:

- $\emptyset \in \mathscr{F}$.

- For all $A, B \in \mathscr{F}$ with $B \nsubseteq A$ and $x=\min _{\leq_{E}} B \backslash A$ we have $A \cup\{x\} \in \mathscr{F}$.

Theorem 2 A (possibly infinite) lattice $P$ is join-distributive and every interval in $P$ is supersolvable if and only if $P$ arises as the lattice of feasible sets of a supersolvable antimatroid.

Our main result is the following. 


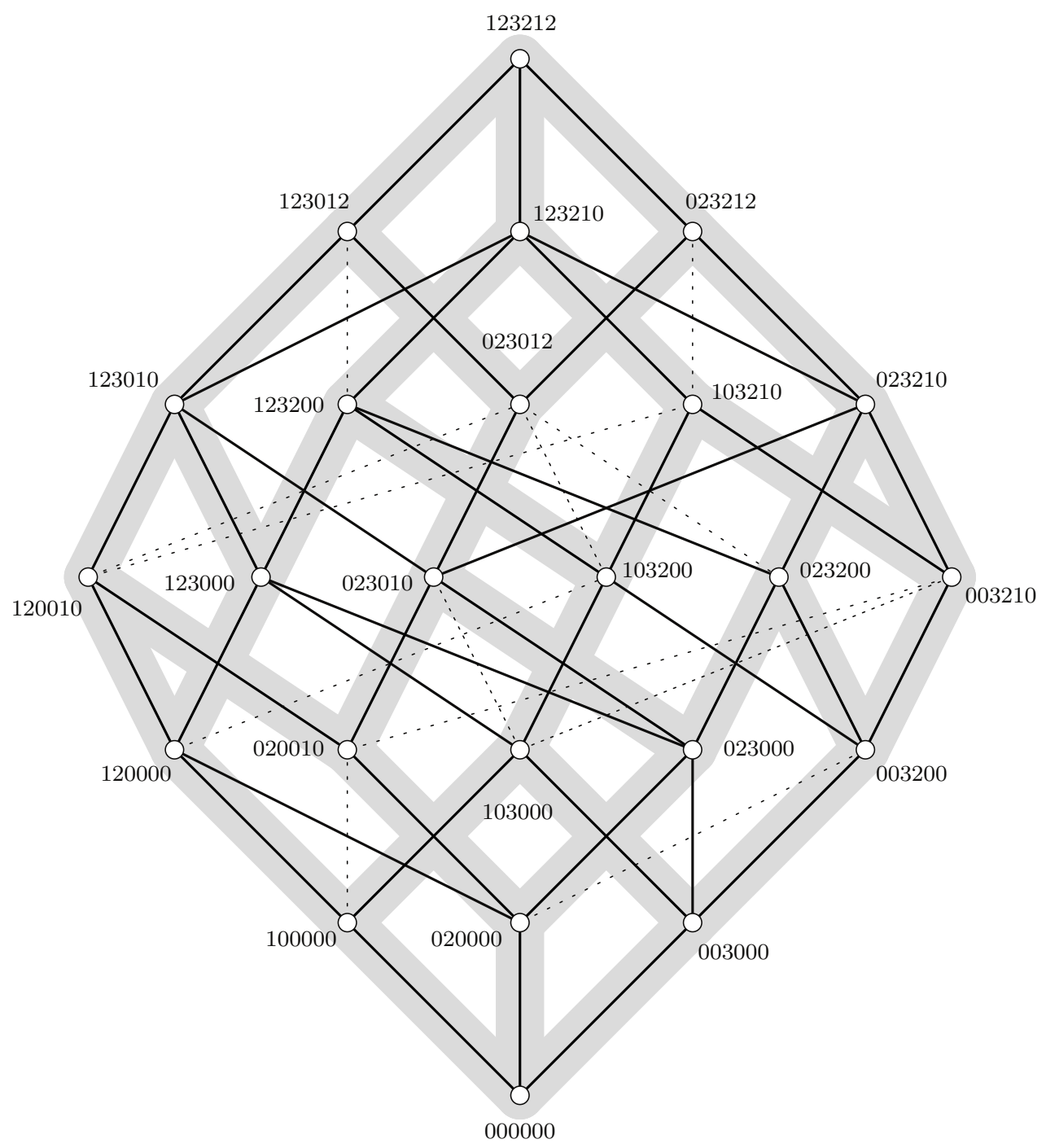

Fig. 1: The weak order, 123212-sorting order and strong order on $\mathfrak{S}_{4}$ 
Theorem 3 Let $(W, S)$ be an arbitrary Coxeter system and consider an arbitrary sequence $\omega \in S^{*}$. The collection of index sets of $\omega$-sorted subwords

$$
\mathscr{F}=\{I(\alpha) \subseteq I(\omega): \alpha \text { is } \omega \text {-sorted }\}
$$

is a supersolvable antimatroid with respect to the natural order on the ground set $E=I(\omega)$.

Corollary 1 The $\omega$-sorting order is a join-distributive lattice in which every interval is supersolvable and it is graded by the usual Coxeter length function $\ell: W \rightarrow \mathbb{Z}$.

Note that this holds even when infinitely many group elements occur as subwords of the sorting sequence. This is remarkable because the weak order on an infinite group is not a lattice. Indeed, we do not know of any other natural source of lattice structures on the elements of an infinite Coxeter group.

We also have

Corollary 2 There exists a reduced sequence $\omega^{\prime} \subseteq \omega$ (that is, every prefix of $\omega^{\prime}$ is a reduced word) such that the $\omega^{\prime}$-sorting order coincides with the $\omega$-sorting order.

That is, we may assume that the sorting sequence is reduced. Finally, we have

Lemma 1 If $\omega$ and $\zeta$ are sequences that differ by the exchange of adjacent commuting generators, then the $\omega$-sorting order coincides with the $\zeta$-sorting order.

In summary, for each commutation class of reduced sequences we obtain a supersolvable join-distributive lattice that is strictly between the weak and Bruhat orders. This is particularly interesting in the case that $\omega$ represents a commutation class of reduced words for the longest element $w_{\circ}$ in a finite Coxeter group.

We end by noting an important special case. Let $(W, S)$ be a Coxeter system with generators $S=$ $\left\{s_{1}, \ldots, s_{n}\right\}$. Any word of the form $\left(s_{\sigma(1)}, \ldots, s_{\sigma(n)}\right)$-where $\sigma \in \mathfrak{S}_{n}$ is a permutation-is called Coxeter word, and the corresponding element $\langle c\rangle \in W$ is a Coxeter element. We say that a cyclic sequence is any sequence of the form

$$
c^{\infty}:=c c c \ldots
$$

The case of $c^{\infty}$-sorted words was first considered by Reading (see $(13 ; 14)$ ), and this is the main motivation behind our work. However, Reading did not consider the structure of the collection of sorted words nor did he consider the sorting order.

\section{Acknowledgements}

The author gratefully acknowledges helpful conversations with Nathan Reading, Vic Reiner, David Speyer and Hugh Thomas.

\section{References}

[1] K. Adaricheva, V. Gorbunov and V. Tumanov, Join-semidistributive lattices and convex geometries, Advances in Math. 173 (2003), 1-49.

[2] D. Armstrong, Generalized noncrossing partitions and combinatorics of Coxeter groups, arxiv:math. Co/ 0611106 , to appear in Mem. Amer. Math. Soc. 
[3] A. Björner and F. Brenti, Combinatorics of Coxeter groups, Springer (2005).

[4] R. Dilworth, Lattices with unique irreducible decompositions, Ann. of Math. 41 (1940), 771-777.

[5] P. Edelman, Meet-distributive lattices and the anti-exchange closure, Algebra Universalis 10 (1980), 290-299.

[6] P. Edelman and R. Jamison, The theory of convex geometries, Geometriae Dedicata 19 (1985), 247270.

[7] G. Grätzter, General lattice theory, Academic Press (1978).

[8] M. Hawrylycz and V. Reiner, The lattice of closure relations on a poset, Algebra Universalis 30 (1993), 301-310.

[9] D. Knuth, The art of computer programming, Volume 1: Fundamental algorithms, Addison-Wesley (1973).

[10] D. Knuth, Axioms and hulls, Lecture Notes in Computer Science, no. 606, Springer (1992).

[11] B. Korte, L. Lovász and R. Schrader, Greedoids, Algorithms and Combinatorics 4, Springer (1991).

[12] P. McNamara, EL-labellings, supersolvability and 0-Hecke algebra actions on posets, J. Combin. Theory Ser. A 101 (2003), 69-89.

[13] N. Reading, Clusters, Coxeter-sortable elements and noncrossing partitions, Trans. Amer. Math. Soc. 359 (2007), 5931-5958.

[14] N. Reading, Sortable elements and Cambrian lattices, Algebra Universalis 56 (2007), 411-437.

[15] D. Speyer, Powers of Coxeter elements in infinite groups are reduced, arXiv: 0710.3188

[16] R. Stanley, Enumerative Combinatorics, vol. 1, Cambridge University Press (1997).

[17] R. Stanley, Supersolvable lattices, Algebra Universalis 2 (1972), 197-217. 
